\title{
First report of pembrolizumab extravasation
}

\author{
Elena Bolzacchini* and Monica Giordano \\ Department of Oncology, Ospedale Sant'Anna, Como, Italy
}

\begin{abstract}
We present an unique case of pembrolizumab extravasation treated with only observation.

This is the first report published so far.

Immunotherapy has completely revolutionized oncology but available guidelines are lacking regarding this topic.
\end{abstract}

\section{To the Editor,}

In the past few years immunotherapy has completely changed treatment strategy of many neoplasms such as melanoma, lung, kidney and head and neck cancer and more recently MSI-high colon cancer, bladder and breast cancer.

Considering the large number of studies ongoing a continuous increase of FDA and EMA indication of immunocheckpoint-inhibitors is expected.

Guidelines regarding immune-related adverse events are very detailed and provide the necessary guidance and recommendations to handle every possible drug related toxicity. [1,2]

On the contrary, available literature about the correct management of immunotherapy extravasation is lacking. Regarding chemotherapy extravasation guidelines are very clear and detailed. [3] Chemotherapy drugs are classified in vescicants, irritants and non-vescicants. The management of extravasations comprehends general actions (stop the infusion, leave the cannula in place, aspirate the extravaseted solution, mark with a pen the extravaseted solution) and specific measures (different antidots).

Immunotherapy extravasation's management is not considered in the available oncological guidelines.

Recently, one of our patients affected with metastatic lung cancer PD-L1 > 90\% was treated with pembrolizumab in first line therapy and he experienced drug-extravasation in our oncological day-hospital.

We applied protocol of extravasation of non-vescicants chemotherapy [3]; specifically, the infusion was stopped, the extravasated solution was aspirated and the area was marked (Figure 1).

According to our internal protocol, plastic surgeon's consult was scheduled, and no specific treatment was suggested.

We observed the patient for 6 hours the same day and the next day. The swollen area disappeared 24 hours after the extravasation.

Current guidelines to not comprehends immunotherapy extravasations.

Specifically, there are not specific recommendations regarding the best and correct management in case of extravasation of nivolumab, pembrolizumab, atezolizumab and durvalumab.

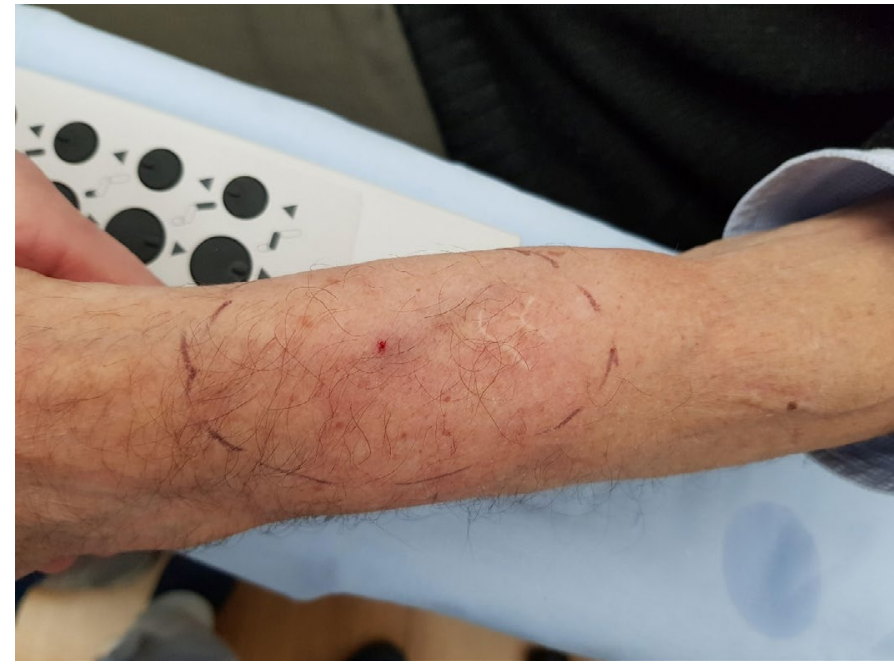

Figure 1. The extravasated solution was aspirated and the area was marked

To the best of our knowledge, this is the first report of an immunotherapy agent extravasation reported in literature. In the absence of clear indications, this complication was handled with only observation, with complete resolution.

Considering the growing number of patients who will be treated with these drugs, clinicians could benefit of specific protocol regarding this important issue, but we report a unique case of pembrolizumab extravasation treated with only observation without consequences.

\section{Author contribution}

The manuscript is approved by all Authors.

All the authors have contributed to the article.

${ }^{*}$ Correspondence to: Elena Bolzacchini M.D, U.O. Oncologia Medica, Ospedale Sant'Anna, ASST-Lariana, Via Ravona 20, San Fermo della Battaglia (Como), Italy, Tel: 00393298597540; E-mail: elena.bolzacchini@hotmail.it

Key words: immunotherapy, extravasation

Received: October 24, 2019; Accepted: November 18, 2019; Published: November 21, 2019 


\section{Conflict of interest}

The authors declare that they have no conflict of interests.

\section{Funding}

No specific funding has been received from any of the authors.

\section{Patients' consent}

Patients' consent was achieved.

\section{References}

1. Brahmer JR, Lacchetti C, Schneider BJ (2018) Management of Immune-Related Adverse Events in Patients Treated With Immune Checkpoint Inhibitor Therapy: American Society of Clinical Oncology Clinical Practice Guideline. J Clin Oncol 36: 1714-1768.

2. Haanen JBAG, Carbonnel F, Robert C (2018) Management of toxicities from immunotherapy: ESMO Clinical Practice Guidelines for diagnosis, treatment and follow-up. Ann Oncol 29: iv264-iv266.

3. Pérez Fidalgo JA, García Fabregat L, Cervantes A (2012) Management of chemotherapy extravasation: ESMO--EONS clinical practice guidelines. Eur J Oncol Nurs 16: 528-534.

Copyright: $@ 2019$ Bolzacchini E. This is an open-access article distributed under the terms of the Creative Commons Attribution License, which permits unrestricted use, distribution, and reproduction in any medium, provided the original author and source are credited. 